Part 2. Reminiscences from Past IAU Presidents 


\title{
The Prague IAU General Assembly, Pluto and the IAU processes
}

\author{
Ron Ekers \\ CSIRO Fellow, IAU President 2003-2006 \\ CSIRO Astronomy and Space Science Australia Telescope National facility \\ P.O. Box 76, Epping NSW 1710, Australia \\ email: ron.ekers@csiro.au
}

\begin{abstract}
At the Sydney IAU General Assembly (GA) the statutes were modified to remove the votes by individual members. Few noticed this at the time but the subsequent reaction by disenfranchised members led us to revise this position in Prague. The need to have a members' vote on the status of Pluto was complicated by these changes and the drama behind the scene at the Prague GA where the planet definition was resolved is not well known despite the huge public impact of this GA. I will describe some of the activities of the executive and its working groups during this very exciting GA. The IAU structures served us well during this process but of course there were also many lessons learned.
\end{abstract}

Keywords. IAU, Prague, Planet Definition, Pluto

\section{IAU structures and policies}

The Statutes \& Bylaws provide the corporate memory of IAU, while the officers rotate continually. The Statutes and Bylaws can only be changed at a GA, so every 3 years. In the period leading up to the IAU GA in Prague the IAU Executive Committee (EC) was implementing new policies to make the IAU more relevant to its members. One item of particular relevance for the Prague GA involved changes to the rules for individual membership. In all Scientific Unions, the primary membership is through the adhering country, but the IAU also has individual membership and for most of the IAU's existence, individual members have been able to vote on scientific issues. However, individual IAU members lost their voting rights after a change to the statutes at the Sydney GA in 2003. After this change, the IAU was criticised for being undemocratic. In making this change in Sydney, the IAU EC had assumed that the National Committees (NC) would consult with the membership and that the $\mathrm{NC}$ votes at the GA would thus reflect the views of the members. However, there were no operating guidelines for the NCs, and big differences in the way the NCs worked in each country. It became clear that there was no consultative process and the $\mathrm{NC}$ vote did not represent the views of the members in each country. IAU members were often unaware of the resolutions to be considered until they arrived at the General Assembly.

The IAU has been the arbiter of planetary and satellite nomenclature since its inception in 1919, and this was one of the reasons for forming such an international body. Science is like an international language. Agreeing on names and conventions is a necessary part of international science, but this is not itself a science. This process is normally handled by the various IAU working groups and their decisions primarily affect the professional astronomers. However, from time to time, the IAU takes decisions and makes recommendations on issues concerning astronomical matters affecting other sciences or the public. Such decisions and recommendations are not enforceable by any national or 
international law, rather they are international agreements on conventions. Any decision that does not have broad consensus will be ineffective.

\section{Changes to our understanding of the solar system}

In 1930 when Pluto was discovered it was assumed to be a few times larger than the Earth but it is now known to be even smaller than the Moon. Many new Trans Neptunian Objects (TNOs) similar to Pluto were also being discovered. One TNO, 2003 UB313, which was found by Mike Brown at Caltech was estimated to be larger than Pluto (Brown et al. 2006a, for example) but no name could be assigned by the IAU because the naming conventions are different for minor solar system bodies and for planets. However, there was, as yet, no planet definition. The IAU had to make a decision on the definition of a planet in order to name the new class of TNOs. Were all the TNOs to be called planets or was Pluto, which all experts agreed was one of the TNOs, to have a special status?

\section{Planetary Definition advisory committees}

The boundary between (major) planets and minor planets has never been defined and the discovery of 2003 UB313, a TNO larger than Pluto, was the trigger for the IAU to act. Following normal procedures, the IAU Executive asked the Planetary Systems Division to form a working group which was to make a recommendation on the "Definition of a Planet". While there was general agreement on all the scientific issues related to the difference between a star and a planet, the solar system dynamics and the physical properties of planets, neither of the IAU Division III working groups could agree on aspects which were related to social and cultural issues such as the status of Pluto. Their recommendations included three options which are summarised here:

(i) Set Pluto as the minimum size - this keeps Pluto a planet and it will add a few other TNOs.

(ii) Include dynamics of the system - this excludes Pluto and Ceres.

(iii) Use round shape (hydrostatic equilibrium) - this keeps Pluto a planet but adds more planets, including Ceres.

In order to include these broader aspects, the IAU Executive Committee formed a new international committee which now also included emphasis on history, outreach, writing and education. It included two active planetary scientists, with the President of IAU Division III being included to represent findings of the earlier Division III working group, and to bring the new committee up to date based on the most recent discoveries.

The Planet Definition Committee Terms of Reference were:

(a) Discuss the broader social implications of any new definition of a planet and recommend a course of action which balances the scientific facts with the need for social acceptance of any change.

(b) Address the status of Pluto, and of the newly discovered TNO's in the light of recommendation (i).

(c) Consider whether the current naming procedures for planets and minor planets have exacerbated the problem of defining a planet and recommend whether revisions are needed.

(d) Attempt to frame these recommendations as a resolution, or resolutions, which can be put before the Prague GA in August 2006 for possible adoption.

According to the IAU Working Rules (IV); "Traditionally, the decisions and recommendations of the Union on scientific and organizational matters of general and significant importance are expressed in the Resolutions of the General Assembly." Any resolution, 
or resolutions, should be framed in such a way that a vote by the IAU individual membership (i.e. a vote by professional astronomers) will be effective. The committee may want to consider resolutions which form a progression of steps so that some progress can be assured even if all aspects are not resolved. Pluto has been with us for 75 years and its status as a planet has been in question for nearly 30 years. We don't have to solve all the problems overnight.

The IAU Planet Definition Committee should report to the IAU General Secretary by 30 June 2006.

\section{Committee membership}

Dr Richard P. Binzel (USA), Professor of Earth, Atmospheric and Planetary Science at MIT.

Dr André Brahic (France), Professor at Université Denis Diderot (Paris VII), and one of the best known popularizers of science and astronomy in France.

Dr Catherine Cesarsky, (Europe) Director. European Southern Observatory and IAU President-elect.

Dr Owen Gingerich (USA) Professor Emeritus of Astronomy and History of Science, Harvard University.

Dava Sobel (USA), the author of the very successful books 'Longitude', 'The Planets', and 'Galileo's Daughter'.

Dr Junichi Watanabe (Japan), Director of the Outreach Division of NAOJ. Highly appreciated in Japan as an interpreter and writer of astronomy for the public and students.

Dr Iwan P. Williams (UK), Queen Mary College, University of London, was the current President of IAU Division III (Planetary Systems Sciences).

\section{The build up to IAU GA in Prague, August 2006}

The IAU EC wanted broad involvement of the IAU membership in the vote on the planet definition. However, individual IAU members had lost their voting rights at the Sydney GA in 2003. The need for reinstating the voting right for individual members was therefore crucial and it had to be done by a change to the statutes during the first business session at Prague so that individual members could vote on the planet definition in the second business session.

Normally, resolutions to be voted on are formulated and made public many months ahead of a GA but, given our awareness of the sensitivity and the risk of derailing the process, we made the decision not to announce anything about the planet definition resolution before the first business meeting of the GA in Prague. Although this ran counter to the need to strengthen the involvement of the membership and the need to conduct all IAU business in an open and transparent manner, we knew that once we made it public there would be strong media interest and pressure to influence the decision-making process.

The IAU Resolutions Committee (chaired by Chris Corbally) was actively involved in the preparation of the resolutions. In anticipation of the public interest we appointed an IAU press officer, Lars Lindberg Christensen from ESO. Lars had been briefed in advance of the Prague GA and, as expected, when the first press release went out the press started pouring in to Prague. They were invited to attend all discussions so that, whilst the preparations had been made discretely, from this stage on everything was played out transparently and in full public view.

The machinery needed for a Prague resolution was now all in place. 


\section{IAU GA 26, Prague 14-25 Aug 2006}

The announcement that there would be a resolution on planetary definitions was included in the President's opening address and was published in detail in the daily IAU newspaper on 16 August 2006. Journalists were informed one day earlier, under embargo. The initial proposed resolution was based on planets being in orbit around the Sun and being round as a result of hydrostatic equilibrium. This would have expanded the number of planets to 12 .

The EC decision to work discretely until the GA caused alienation of the general scientific community, which was regrettable and effected the vote ("let's punish the IAU by voting against their proposal"). However, had we gone public earlier there would have been even more impact from the issues triggered by Pluto's status. Holding the debate at the GA in public had a huge and positive impact on all except some planetary scientists who were not attending this GA and felt excluded from the action.

Vigorous open discussions with all IAU participants took place in a special Plenary Session and more discussions took place in the Divisional meetings. In these discussions some strongly held views emerged.

(a) Planetary definition committee view: keep it simple, using round shape and the classical "planet" category. However, the need to exclude planetary satellites (e.g. the Moon) resulted in a footnote that complicated this simple case.

(b) Solar system formation and planetary dynamics view: Massive objects can clear their orbit and this complementary aspect of planetary systems must also be considered. Because of the associated symposium on Planetary Dynamics (S236), we had a relatively larger number of planetary dynamicists at this GA.

(c) Planetary geologists view: Shape and structure are all that matters, names should not depend on the environment.

(d) Should exoplanet issues be included? The IAU EC had no formal request to do so at this stage from either the Exo-planet Commission or the Commission on Nomenclature.

The resolution was then modified in consultation with the IAU Resolutions Committee and the Planetary Systems Division in response to their feedback and to a straw poll which indicated that the initial version of the resolution would not be passed. If not passed that would mean that the Planetary definition issues would not be resolved at this IAU GA and it would be a further three years to the next GA.

Obviously, the continual redrafting of resolutions during the GA was less than optimal, but it did demonstrate an active process of consultation. The modified multi-part resolution was then voted on by those astronomers present in the final session of the GA. For maximum transparency, the discussions and final vote were open to the press but only IAU members were issued with yellow voting cards.

\section{The IAU Resolutions}

\section{IAU resolution $5 \mathrm{~A}$ :}

(a) A planet is in orbit around the Sun, has sufficient mass to assume hydrostatic equilibrium (nearly round) and has cleared the neighbourhood around its orbit.

(b) A dwarf planet is also round but doesn't dominate its neighbourhood and is not a satellite.

Resolution 5A passed comfortably with no need for a count, so Pluto became a "dwarf planet".

IAU resolution 5B: Classical and Dwarf planets are all planets.

This resolution would have left Pluto and the other dwarf planets in a broader, more inclusive, class of planets. With only 91 votes in favour, this vote failed by a significant 


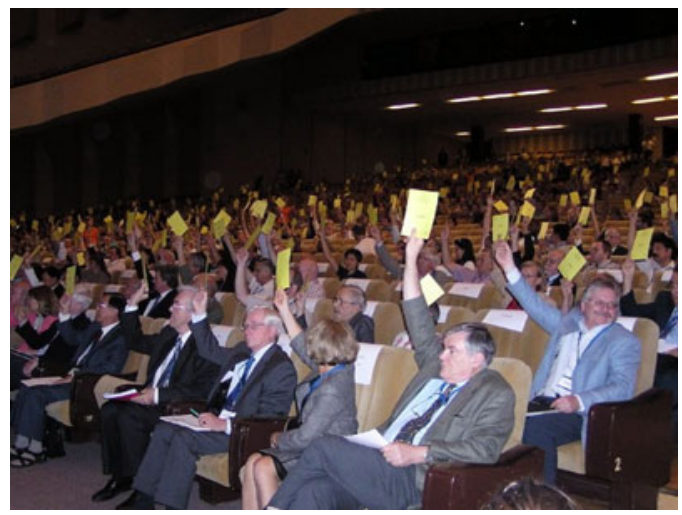

Figure 1. The vote on resolution 5A, IAU GA Prague.

margin, so a Dwarf planet is not a planet and at 15:34 CEST 24 Aug 2006 Pluto ceased to be a planet as defined by IAU resolutions.

IAU resolution 6A: Define a new Pluto-class of objects, recognising Pluto's historical significance.

Passed 237 in favour, 157 against (17 abstentions).

\section{$I A U$ resolution 6B: The new class are called Plutonians}

The preferred name proposed by the Planet Definition Committee had been Plutons but there was strong resistance from the geologists, since pluton is a type of rock. This was an unfortunate consequence of the lack of open discussion with other scientific bodies before the Prague GA. The name plutons was withdrawn by the EC and the resolution was changed to plutonians at short notice.

This vote failed but was very close - 183:186. The objection was that it sounds too close to plutonium. but it is ironical to note that uranium was named chronologically after Uranus and plutonium was named after the planet Pluto! No name was assigned at the Prague GA but the name plutoids was later adopted by the IAU Executive Committee at its meeting on 11 June 2008.

\section{Dwarf Planets}

With the planet definition settled, 2003 UB313 is a dwarf planet (the largest known) and was appropriately named Eris by its discoverer Mike Brown, Eris being the goddess of strife and discord! Brown et al. (2006a) discovered a moon orbiting Eris and this observation gave a mass estimate confirming that Eris is $27 \%$ more massive than Pluto. By 2012 there were five Dwarf Planets named by the IAU: Ceres, Pluto, Eris, Makemake and Haumea.

\section{Outreach activities at the Prague GA}

Lars Lindberg Christensen ran the Press office at the GA and was well prepared to handle the expected media interest. The perceived need by the press office to make the resolutions clear to the public did make it look like the IAU EC had an agenda rather than conducting an open debate, but the activities of the press office were essential to maintain the open public communication during the GA. A full report of the public communication activities at the Prague GA is available from the IAU web-site www.eso.org/lchriste/trans/pluto.

Global press coverage was unprecedented, and this included coverage of all astronomy and not just Pluto and the planet definition. The public knowledge of the IAU today has been greatly enhanced by this GA. 


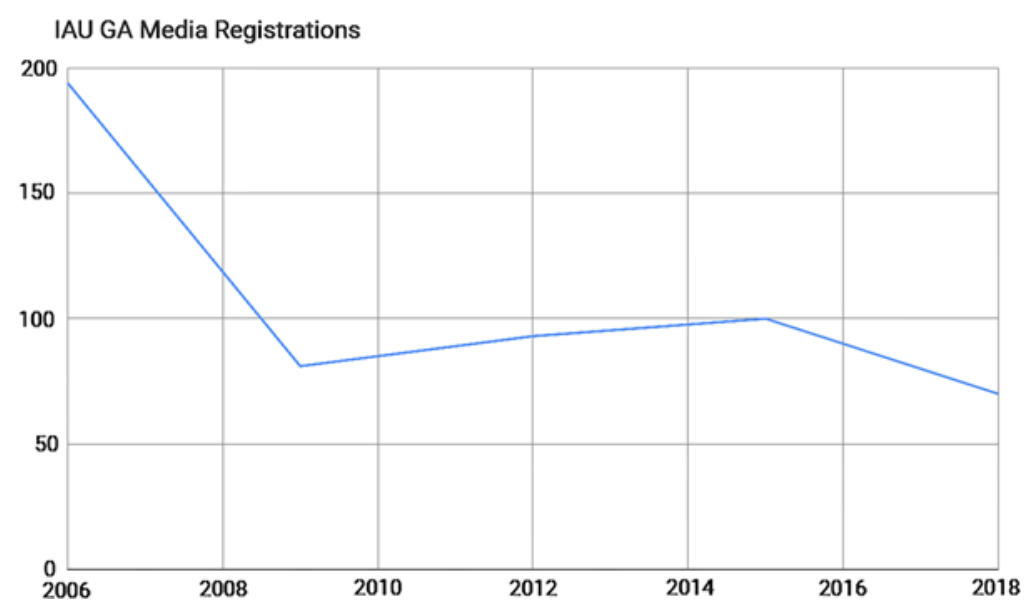

Figure 2. Number of media registrations at IAU GAs for last five GAs.

\section{New Horizons}

The New Horizons space mission was launched by NASA on 19 Jan 2006 to conduct a six-month-long reconnaissance fly-by study of Pluto and its moons. It reached Pluto nine years later, culminating with Pluto's closest approach on 14 July 2015. The IAU resolution to re-classify Pluto as a dwarf planet was taken while New Horizons was en route to Pluto. Obviously, the IAU resolution was not relevant for this remarkably successful mission, but now we can say that New Horizons was not only exploring the first TNO but also discovering how spectacularly different are the Dwarf Planets.

\section{Epilogue}

The IAU planet definition has had a large impact and while there was, and still is, a vocal minority, the general reaction has been positive. Here are a few examples:

- Neil deGrasse Tyson had not included Pluto in the planetary display of the Hayden Planetarium, pre-empting the IAU resolution by many years.

- Daniel Fischer (Fischer 2006) provided the most detailed and accurate account of the activities at the Prague GA. He concluded by saying "The procedural flaws in the handling of the whole affair by the IAU EC are rather obvious, especially the secrecy prior to August 16 that backfired by compressing the whole complex debate into a window of eight days, and the overly hasty drafting of resolution 5 a that had to follow. Nonetheless, during the debates on August 18 and later, all the key arguments for the various possible planet definitions were presented already... Then, a cross-section of the world's astronomers which a dedicated anti-IAU conference would hardly bring together in such a way - held a vote, with a clear result which in turn is already gaining some acceptance because the 'concept', to use Mike Brown's words, is sound."

- Burial services were held at some public science centres and used to explain the way science progresses (Crider 2011).

- Role playing simulations for students which include the IAU planet debate.

- A simple metric that quantifies "has cleared the neighbourhood around its orbit" and extends the definition to planets orbiting other stars has now been published by Jean-Luc Margot (Margot 2015).

- Mike Brown (Brown et al. 2006b) "Because of the relatively chaotic process that occurred before reaching this very rational decision, the actual wording of the definition is not as precise as it might have been, giving people room to quibble and to say that the 
definition is unclear. The important point to remember, however, is that the difference between the eight planets and everything else known in the solar system is so huge that even a definition with a lot of wiggle room will not make any difference".

And there were other less positive reactions but still typical of the broad community engagement:

- February not a month, Michael Haber (Haber 2006). "Emboldened by their success in declaring Pluto not a planet, the International Astronomical Union determined this week by a close vote that February is too short to be considered a true month. It has, however, been granted the newly created status of "dwarf month'."

- Legislation in New Mexico and Illinois in the US restored Pluto's planetary status in these states.

- No astrologers were included on the planet definition committee!

- "Plutoed" was chosen 2006 Word of the Year by the American Dialect Society, www.americandialect.org/plutoed_voted_2006_word_of_the_year

The need for electronic voting was recognised and included in the IAU Statutes as a result of the Pluto debate. This process will be used for the Hubble vs Hubble-Lemaitre resolution in 2019 .

\section{References}

Brown, M. et al. 2006a, Ap.J., 639: L43-46

Brown, M. et al. 2006b, web.gps.caltech.edu/mbrown/eightplanets

Crider, A., 2011. Debating Pluto: Searching for the Classroom of the Future and Ending up in the Past. Astronomy Beat, 74(1).

Fischer, D. 2006, Inside the planet definition process, The Space Review, Monday, September 11, 2006

Haber, M. (2006) Astronomers Declare February No Longer a Month, Submitted to a joke website gcfl.net/archive.php?funny $=4775$ and widely circulated on the www

Margot, J.-L. 2015, A Quantitative Criterion for Defining Planets, A. J., 150, issue 6, id 185, 7pp 